\title{
clouage des sols : règles de dimensionnement et leur vérification expérimentale ${ }^{*}$,
}

\author{
par \\ G. Gudehus \\ Professeur de Mécanique des Sols et Fondations \\ I. B. F. Universität Karlsruhe
}

\section{Introduction}

Par clouage (cloutage) on entend le renforcement du sol in-situ à l'aide de barres raides (Louis, 1981). Le schéma d'exécution typique est montré sur la figure 1. On commence par une excavation d'environ 1 à $1,5 \mathrm{~m}$ de profondeur (a). Le sol doit avoir une certaine cohésion, soit-elle seulement capillaire, pour être suffisamment stable lors de cette étape. La nouvelle surface est couverte par un revêtement (b). Cela consiste, dans la plupart des cas, en une coque de béton projeté, renforcée par un réseau d'acier. Une rangée d'armatures est alors montée (c). Normalement des barres en acier sont placées dans des trous forés et scellées avec du ciment. Leur longueur est de 3 à $8 \mathrm{~m}$, le diamètre externe allant de 7,5 à $15 \mathrm{~m}$, et la distance horizontale pouvant varier entre 1 et $2 \mathrm{~m}$.
Une étape suivante plus profonde est exécutée de la même manière. Pendant l'excavation le sol est déjà stabilisé par les armatures supérieures (fig. 1 d). Si la cohésion du sol est trop faible on peut réduire la hauteur du pas. Le revêtement est appliqué comme auparavant (e). Puis, les armatures sont placées, leur longueur croissant normalement avec la profondeur (f). Dans le cas de murs presque verticaux, on peut atteindre une profondeur totale de 15 à $20 \mathrm{~m}$, selon les caractéristiques du sol (voir paragraphe 3.3).

Si la surface du sol a une forme différente, l'arrangement des armatures doit être changé (fig. 2). Dans un terrain en pente faible on peut installer des barres verticales traversant les surfaces de glissement potentielles (a). La capacité portante d'un sol mou peut être augmentée par des armatures inclinées (b). Le

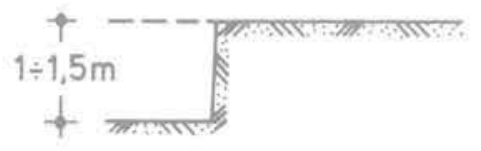

a)

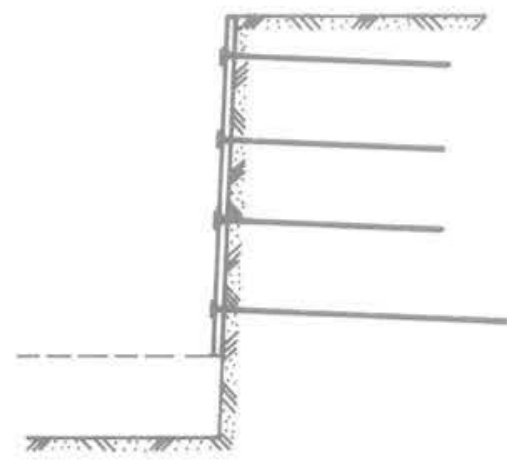

d)

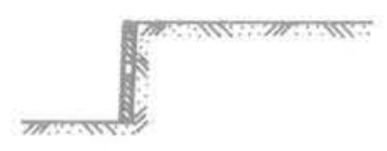

b)

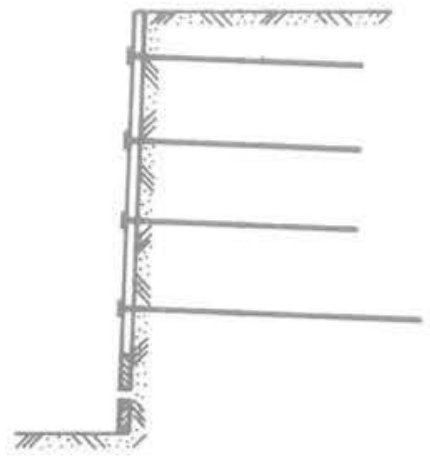

e)

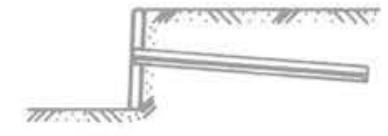

c)

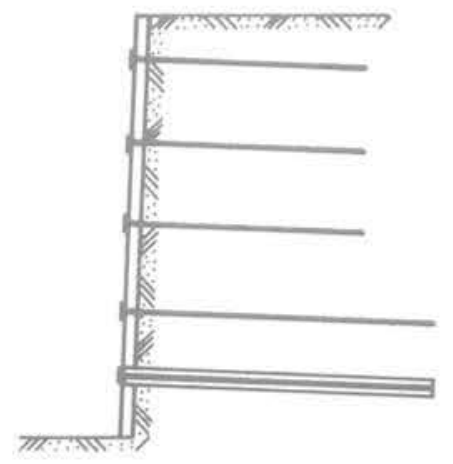

f)

Fig. 1 Phases d'exécution :

a) 1ère excavation $-b$ ) 1er revêtement $-c)$ 1er renforcement - d) nième excavation - e) nième revêtement - f) nième renforcement

(") D'après une contérence donnée au Comité Français de Mécanique des sols, Paris, le 18 mai 1981. 


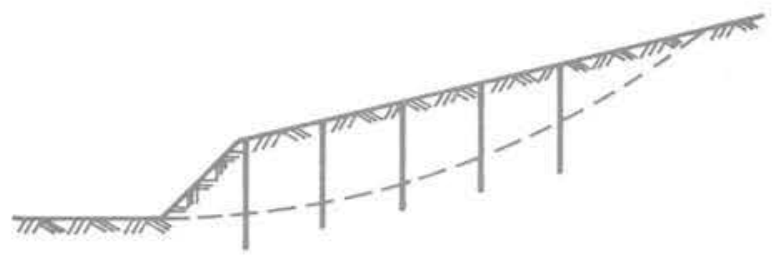

a) le long d'un talus

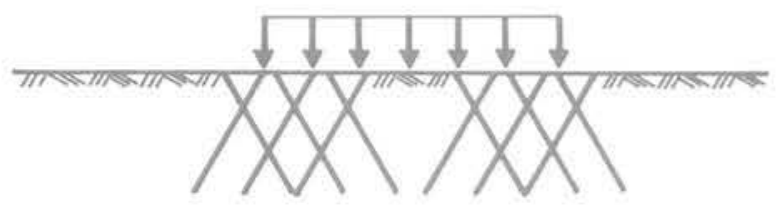

b) dans un sol mou

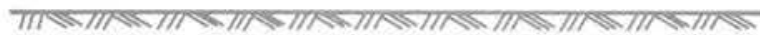

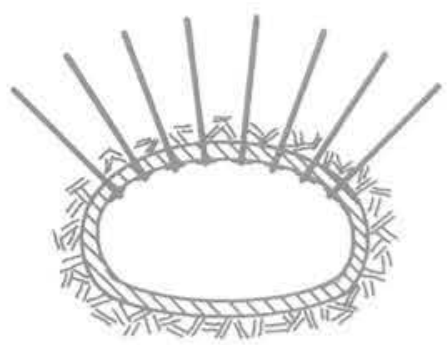

c) autour d'un tunnel

Fig. 2 Arrangements des armatures

massif de terre autour d'un tunnel peut être stabilisé par des ancrages sans précontrainte (c). Le dernier système est bien connu en relation avec la méthode nouvelle autrichienne.

Un projet de recherche et développement sur le clouage a été effectué durant les années 1975 à 1980 en Allemagne. Pour augmenter et vérifier le savoir technologique, l'entrepreneur K. Bauer (le même que celui qui a développé des techniques d'ancrage dans les sols depuis presque 30 ans) a effectué neuf essais à grande échelle. Le IBF Karlsruhe a développé des règles pour le dimensionnement et les a vérifiées par des mesures in situ aussi bien qu'en laboratoire. $\mathrm{Ce}$ projet était partiellement financé par le Ministère Fédéral de la Recherche et de la Technologie (BMFT). Entre-temps la Société Bauer a réalisé plus de vingt projets réels avec le même procédé.

Le présent rapport est principalement consacré aux méthodes de dimensionnement et à leur vérification. Dans le paragraphe 2, on traitera du cas des sols granulaires. Les sols argileux seront l'objet du paragraphe 3 . Un projet réel dans un sol mixte sera décrit dans ce contexte. Finalement quelques développements pratiques et théoriques récents seront brièvement traités (paragraphe 4).

\section{Sols granulaires}

Les buts du dimensionnement sont d'assurer, dans l'ordre d'importance suivant, la stabilité globale et locale, et la petitesse des déformations. On expliquera d'abord les méthodes théoriques développées pour cela à Karlsruhe, et ensuite leur vérification expérimentale. L'exposé suivant se restreindra au système de la figure 1 .

\section{1 Études théoriques}

La stabilité globale se perd par le glissement de prismes de terre. Diverses surfaces de glissement sont cinématiquement possibles (fig. 3). Dans le cas d'une suriace de Coulomb (plane ou faiblement courbée) on aura un seul prisme, et les armatures inférieures sont arrachées (a). Un cercle de glissement profond peut entourer tous les barres et sortir devant le pied (b). Un prisme de terre peut pousser latéralement un autre prisme sur un plan de glissement moins incliné que la pente du prisme supérieur. Lors d'un tel mécanisme composé on aura une troisième surface de glissement séparant deux corps rigides (Gudehus, 1972). Les deux corps peuvent aussi être formés par des surfaces cylindriques (d). Dans le cas d'un tel mécanisme de rotation les trois centres des cercles doivent être sur une droite.

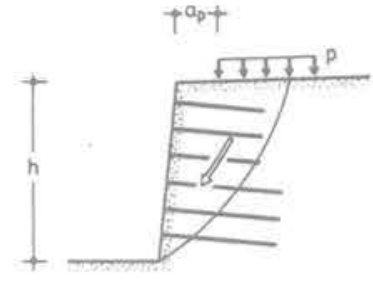

a)

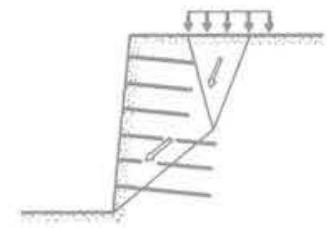

c)

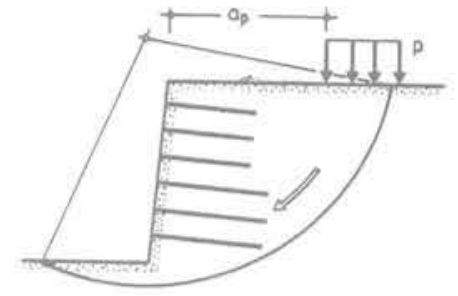

b)

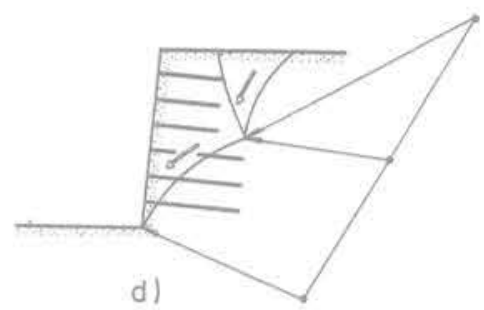

Fig. 3 Mécanismes de ruine d'un massif clouté :

a) prisme de Coulomb

b) cercle de glissement profond

c) translation composée

d) rotation composée

L'analyse statique suit les principes bien établis de la Mécanique des Sols. Les forces agissantes par unité de longueur sur un prisme de Coulomb sont, à titre d'exemple (fig. 4a):

W. poids:

$P$. surcharge résultante;

Q, résultante de la force normale et de la force de frottement associée, inclinée à $\varphi$ par rapport à la normale;

A. force axiale résultante des parties arrachées des armatures.

La force $A$ est calculée par

$$
A=T_{m} \Sigma l_{\text {ia }}
$$


$T_{m}$ étant la force d'arrachement par unité de longueur

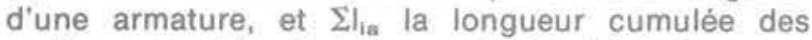
sections arrachées (relativement à l'unité de longueur perpendiculairement au plan de représentation). L'équilibre d'état limite peut être atteint par des causes diverses. Par exemple, la surcharge $P$ peut croître sans changement des autres variables (fig. 4 b). Ou bien, la force $A$ peut décroître sans variation de $P$, ou bien l'angle de frottement peut être réduit. Pour obtenir un seul facteur de sécurité on peut introduire une force fictive $T_{\text {t }}$ agissante le long de la surface de glissement (Goldscheider et Kolymbas, 1980). T, doit remplacer les diverses causes de ruine (réduction de $\varphi$ ou $T_{m}$. augmentation de $\mathrm{P}$ etc.). Le facteur global de sécurité est défini par

$$
\eta:=\frac{T_{A}}{T_{A}} .
$$

$T_{A}$ symbolise le travail virtuel des forces résistantes $Q$ et $A$ calculées avec la force fictive $T_{f}$ (fig. $4 \mathrm{c}$ ), tandis que $T_{A}$ représente le travail des forces motrices $W$ et $P$. Le déplacement virtuel est un glissement du prisme.

$W, P, A$ et $\eta$ sont fonctions de la pente $\theta$ du prisme. Généralisant le principe de Coulomb, on doit faire varier $\theta$ pour obtenir le minimum de $\eta$. On peut appeler $\theta_{a}$ la position du minimum; sa valeur est simplement appelée $\eta$ parce que cet $\eta$ seul est déterminant pour le dimensionnement.

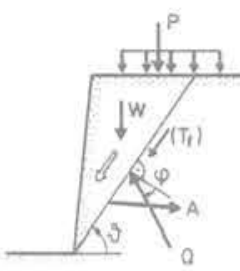

a)

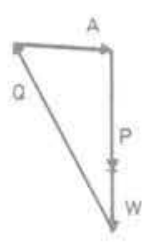

b)

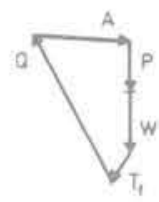

c)
Fig. 4 Analyse statique d'un prisme clouté : a) système et forces

b) équilibre limite avec surcharge augmentée

c) équilibre limite avec effort tranchant fictif

Le même principe de la sécurité minimale (Gudehus, 1981) s'applique aussi pour les autres mécanismes de la figure 3 . On a besoin de plusieurs variables cinématiques pour décrire le mécanisme

a) 2 pour le cercle à travers le pied du mur,

b) 3 pour le cercle profond,

c) 3 pour la translation composée,

d) 5 pour la rotation composée.

En introduisant une force fictive $T_{\text {, }}$ on peut satisfaire les conditions d'équilibre limite. Comme dans le cas de la figure $4, T$, doit remplacer un changement défavorable des paramètres de charge et de résistance. C'est pourquoi le facteur de sécurité $\eta$ et la position de son minimum dépendent du choix de $T_{f}$ (voir paragr. 4.2).

Pour un certain ensemble de paramètres (de géométrie, matériaux et charge) on peut déterminer le mécanisme le plus défavorable dans le cadre de la figure 3. Par une série de calculs comparatifs on peut démontrer :

a) que le mécanisme de Coulomb n'est déterminant que si la charge est forte (environ $p>y h / 2$ ) et proche du bord (environ $\mathrm{a}<\mathrm{h} / 4$ ); b) que le cercle profond peut être déterminant dans le cas exceptionnel d'une charge très forte et éloignée du bord;

c) que la translation composée est la plus probable (i.e. $\eta$ est minimal) dans la plupart des cas réels (environ $p<\gamma h / 2, a>h / 4$ );

d) que la rotation composée donnant le minimum de $\eta$ est très proche de la translation, de sorte que ce cas ne vaut pas la peine d'une analyse.

La stabilité locale ne peut encore être estimée avec la même précision. Dans le cas de conditions extrêmes il est possible que la coque ou les armatures soient déchirées. La distribution des efforts le long des parties de la structure dépend de leur déformation relative par rapport au sol d'une manière très compliquée. De même une prédiction exacte des déplacements est encore impossible.

Cependant, les lois de similitude pour des modèles en sable permettent d'établir une approximation grossière. Pour des contraintes modérées (environ $\alpha<$ $1 \mathrm{MPa}$ ) le sable est complètement décrit par des caractéristiques sans dimensions (Gudehus, 1980 a). C'est pourquoi on peut transférer toute quantité sans dimension mesurée dans un modèle (Gudehus, 1980 b). Pour le clouage d'un déblai dans l'état en service les quantités suivantes sont importantes (fig. 5) :

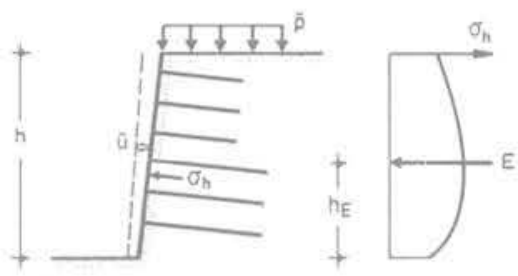

Fig. 5 Quelques quantités déterminantes dans l'état de service

a) $\bar{u} / h, \bar{u}$ étant le déplacement moyen du mur. Selon la densité on aura

$$
\mathrm{u} / \mathrm{h}=0,001 \text { à } 0,003 \text {. }
$$

b) $K$, coefficient de pression des terres, défini par

$$
E=\left(\frac{1}{2} \gamma h^{2}+\bar{p} h\right) K \text {, }
$$

$E$ étant la pression de terre et $\bar{p}$ la surcharge moyenne sur le prisme de Coulomb. On aura

$$
\mathrm{K}=0,5 \mathrm{~K}_{\mathrm{a}} \text { à } 0,6 \mathrm{~K}_{\mathrm{a}} \text {, }
$$

$\mathrm{K}_{\mathrm{a}}$ étant le coefficient de poussée des terres. (On néglige ici la cohésion capillaire.)

c) $h_{E} / h, h_{E}$ étant la hauteur du point d'application de la résultante $E$. On aura

$$
h_{E} / h=0,4 \text { à } 0,5
$$

selon que la surcharge est faible (environ $\bar{p}<\gamma h / 2$ ) ou non.

Ces indications suffisent pour estimer les efforts dans la coque et les armatures. On doit les augmenter par des facteurs de sécurité purement empiriques pour le dimensionnement. 


\subsection{Vérification expérimentale}

Par plusieurs essais à échelle réduite on a vérifié principalement les mécanismes de ruine. On a fait varier l'arrangement des armatures et la position de la surcharge. La force portante $T_{m}$ par unité de longueur des barres était réduite de sorte que

$$
T_{m} / \gamma h^{2} \approx \text { const. }
$$

avec la même constante dans le modèle et le prototype. (La dépendance de $T_{m}$ de la profondeur était faible et négligeable par cette raison). Le sable était mis en place par pluie en chute libre de façon à obtenir diverses densités.
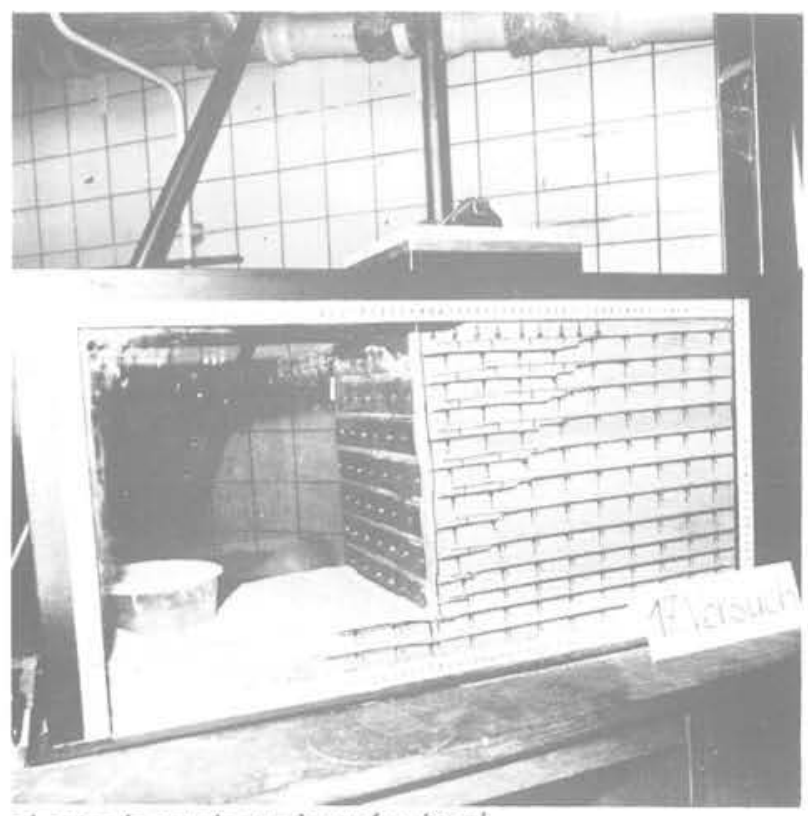

a) avec la surcharge jusqu'au bord

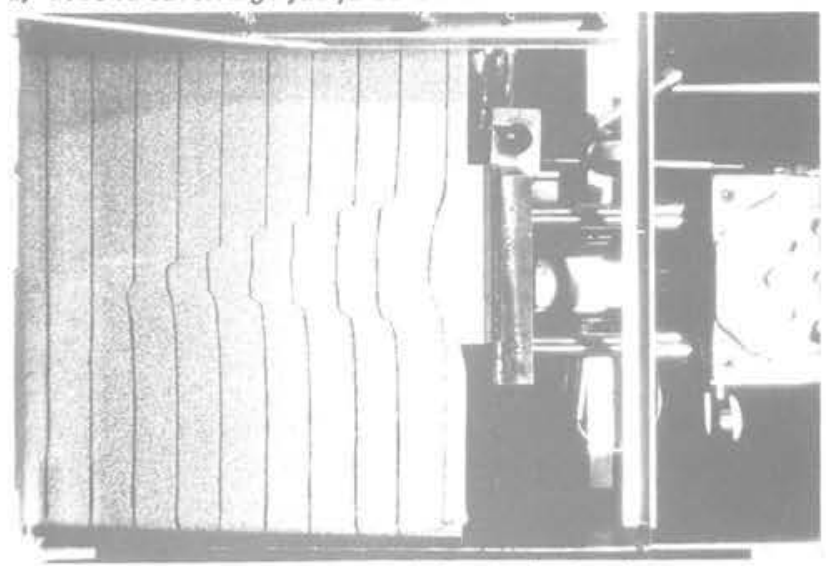

b) avec une surcharge assez éloignée du bord

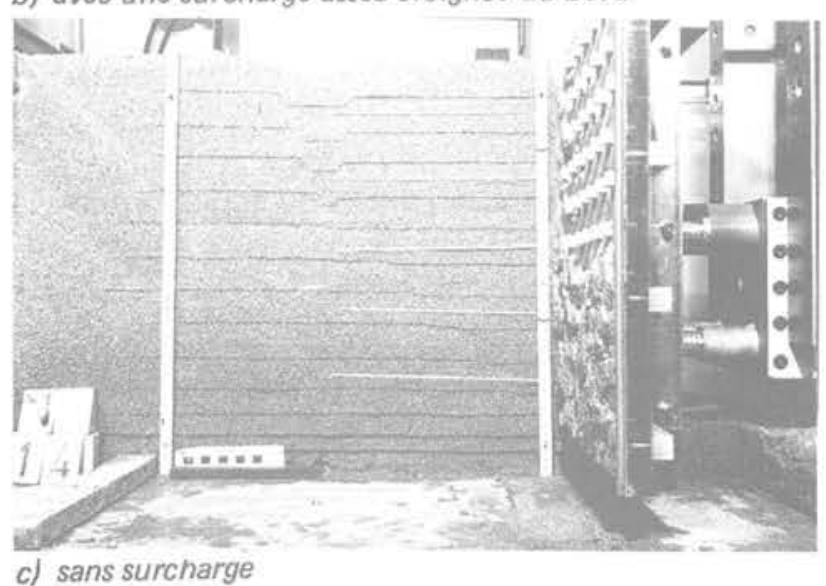

Fig. 6 Mécanismes de ruine observés à échelle réduite
La figure 6 montre trois mécanismes de ruine fréquemment observés. Avec une surcharge atteignant le bord on obtient le mécanisme de Coulomb (a). Les détails de cet essai sont décrits par Stocker et al. (1979). Dans le cas d'une surcharge plus éloignée du bord on obtient une translation composée (b). Le même mécanisme est aussi provoqué par un déplacement du mur sans surcharge du sol (c). Pour plus de détails voir Gässler (1982).

Quatre essais à grande échelle ont été exécutés dans une formation de sable fin de densité moyenne. Le dispositif de deux essais est montré sur la figure 7 . Pour obtenir une déformation plane dans une section de $7 \mathrm{~m}$ de longueur, deux couches de glissement ont été arrangées jusqu'à une profondeur suffisante. Grâce à ces couches, constituées par $4 \mathrm{~cm}$ de bentonite, les déplacements furent en effet limités à l'espace compris entre elles. Les charges ont été appliquées par des poutres et des presses hydrauliques qui s'appuyaient sur des ancrages très profonds. A deux emplacements différents on a augmenté la surcharge jusqu'à l'état limite. Une vue totale de l'essai $n^{\circ} 2$ est montrée sur la figure 8 .

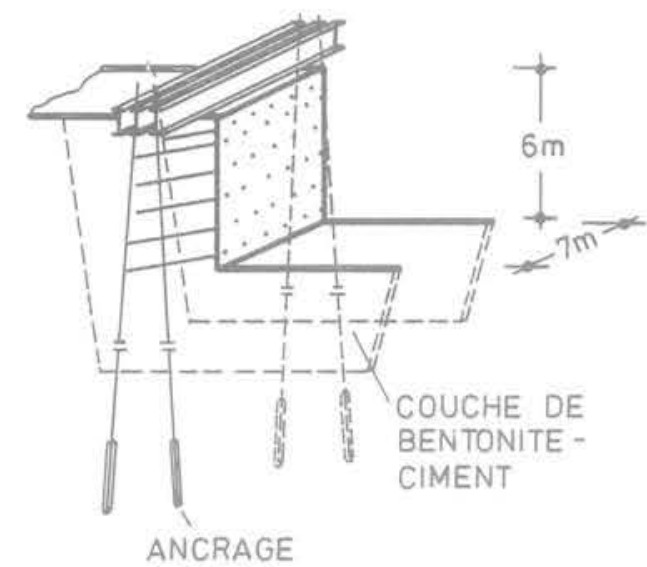

Fig. 7 Dispositif des essais à grande échelle en déformation plane

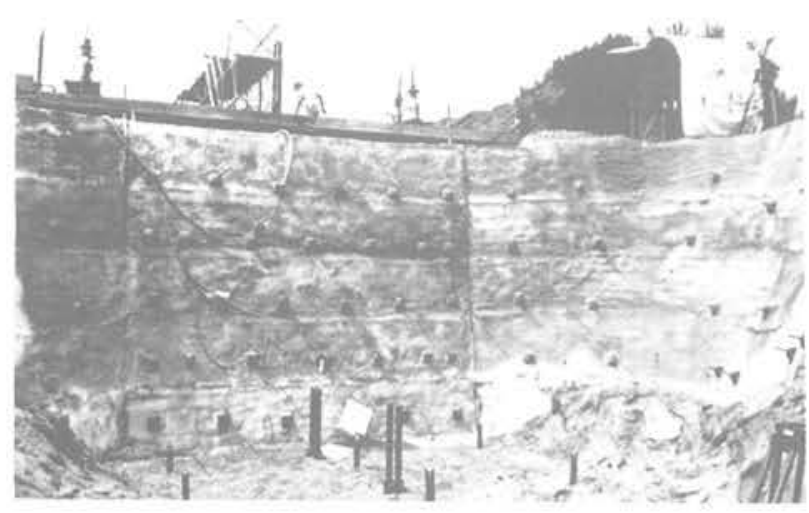

Fig. 8 Essai à grande échelle dans un sable fin 
Les déplacements ont été mesurés à la surface et dans l'intérieur du sol, ces dernières à l'aide d'inclinomètres dans 5 trous forés. Les mécanismes de ruine observés sont montrés sur la figure 9 . Lors du premier essai on n'a pu réaliser la ruine qu'après avoir éliminé la rangée inférieure des clous et approfondi l'excavation (a). Ceci est clairement le mécanisme de la figure $3 \mathrm{c}$. Le mécanisme de la figure $3 a$ a été réalisé par une surcharge plus proche au bord (b). Par une déformation continue selon le même mécanisme le sol a atteint l'état critique dans les surfaces de glissement. Les conditions d'équilibre sont satisfaites avec l'angle de frottement résiduel et les forces des armatures selon l'équation 1.

Les efforts axiaux dans les armatures pour le cas de la figure $9 \mathrm{~b}$ sont montrés sur la figure 10. En gros ils croissent linéairement dans les sections finalement arrachées. La pente moyenne des courbes à droite est égale à la force $T_{m}$. La même pente environ a été trouvée lors d'un essai d'arrachement avec un clou isolé (Gässler et Gudehus, 1981). Les efforts axiaux décroissent vers le revêtement.

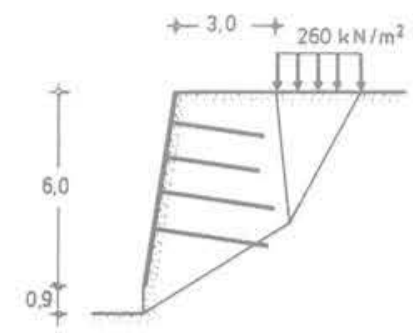

a)

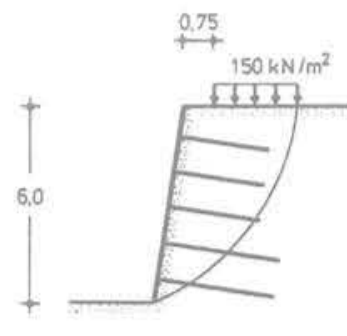

b)
Fig. 9 Mécanismes de ruine observés lors de deux essais à grande échelle

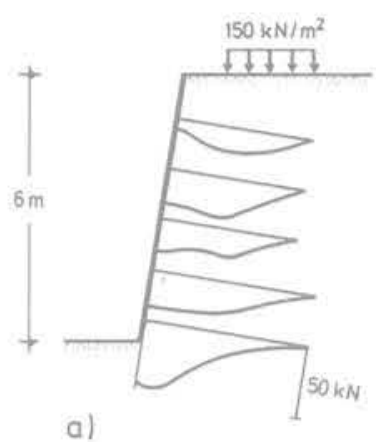

Fig. 10 Efforts axiaux le long des armatures

Les déplacements horizontaux du revêtement lors de l'excavation ont atteint environ $\widetilde{u} \simeq 0,002 \mathrm{~h}$ en décroissant avec la profondeur. Sous la surcharge maximale ils ont crû jusqu'à $\overline{\mathrm{u}} \approx 0,004 \mathrm{~h}$ avec leur maximum au milieu de la hauteur. D'autres détails sont décrits par Stocker et al. (1979) et Gässler et Gudehus (1981).

La figure 11 donne un exemple des pressions des terres mesurées par des cellules Glötzl. La pression résultante coïncida avec celle mesurée en tête des armatures. Après l'excavation la distribution est presque linéaire (a). D'après les équations 4 et 5 , on obtient $K \approx 0,5$. Sous la surcharge les pressions croissent particulièrement vers le pied du revêtement (b). La résultante peut être calculée par l'équation 4 avec $\mathrm{K} \approx 0,65$.
Le troisième essai à grande échelle a été exécuté avec une surcharge partiellement cyclique. Des sollicitations équivalentes à celles exercées par de lourds camions ont été appliquées par un pulsateur hydraulique. Le sol cloué a montré une adaptation marquée indiquant que les charges cycliques réelles ne mettent pas en danger la structure. Les détails sont décrits par Gässler (1978).

Lors d'un autre essai à grande échelle on a chargé un coin convexe. Dans ce cas tridimensionnel la densité et la longueur des armatures étaient les mêmes que dans les essais "plans" (selon la fig. 7). La figure 12 montre le système après l'application de la charge maximale. Quoique la coque soit fendue, il n'y a pas eu d'effondrement général. Pour les détails voir Stocker et Gässler (1979).

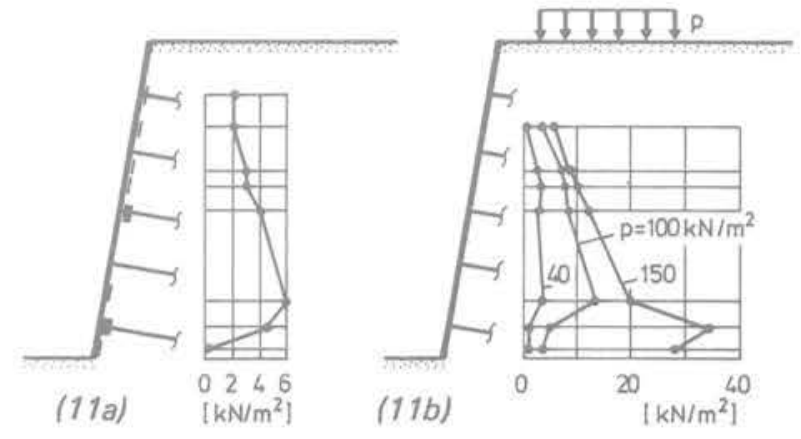

Fig. 11 Pressions des terres mesurés :

a) après l'excavation

b) sous la surcharge

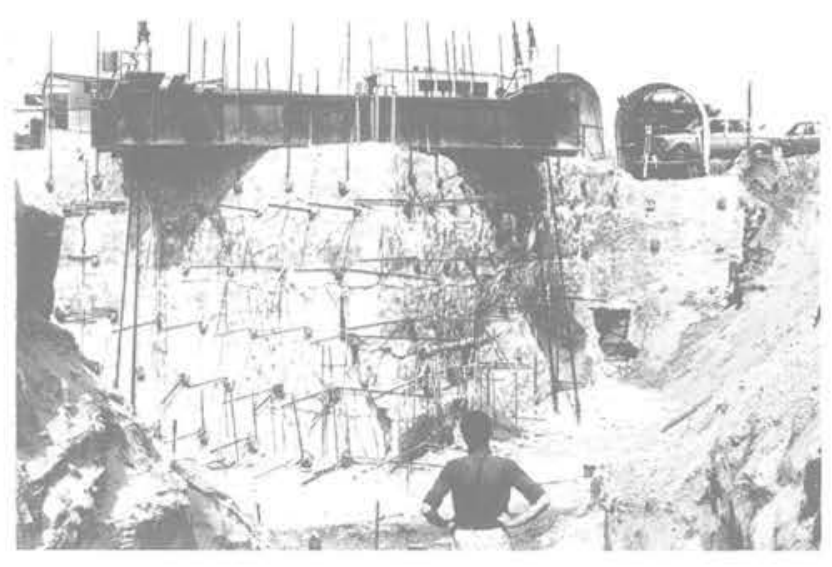

Fig. 12 Coin convexe après chargement jusqu'à l'état limite

\section{Sols argileux}

Comme c'est I'habitude en mécanique des sols (Gudehus 1981), on peut distinguer trois types de sols argileux, à savoir

- ceux sans préchargement, mous de ce fait (traités dans le paragraphe 3.1);

- ceux préalablement chargés, raides de ce fait (paragraphe 3.2);

- les sols argileux raides avec fissures et cimentation (paragraphe 3.3).

La théorie n'est pas encore aussi bien développée que pour les sols granulaires. C'est pourquoi nous nous concentrerons ici sur les résultats expérimentaux. 


\subsection{Argiles non surconsolidés}

Le but du clouage d'un sol mou est - d'après la fig. $2 \mathrm{~b}$ - d'augmenter la capacité portante et d'égaliser les tassements inévitables. Un mécanisme possible de ruine est une rotation avec un cercle de glissement profond (fig. 13). Évidemment la sécurité est augmentée par les efforts tranchants (appelés T) des armatures cisaillées. Les barres fonctionnant comme des goujons, on peut parler aussi d'un goujonnage. L'arrangement du type de la fig. $2 b$ résulte du calcul de ces cercles de glissement qui donnent une sécurité insuffisante sans des forces T. A partir de la théorie d'écoulement autour d'un pieu on peut s'attendre à ce que T croisse avec l'épaisseur de la barre, la cohésion non drainée du sol et la vitesse de déformation (Winter 1980).

On a étudié divers arrangements des armatures par des essais à échelle réduite et des essais de cisaillement. Deux essais à grande échelle ont été exécutés, l'un dans une argile lacustre et l'autre dans une argile organique avec des couches minces de sable fin. Les armatures consistaient en des palplanches légères enfoncées par pression et ébranlements (fig. 14). Des remblais de $5 \mathrm{~m}$ ou de $3 \mathrm{~m}$ de hauteur avaient été disposés sur les zones armées, et sur le sol voisin non armé. On a observé des tassements réduits et plus uniformes grâce aux armatures (Gudehus 1979). Ce clouage est assez coûteux.

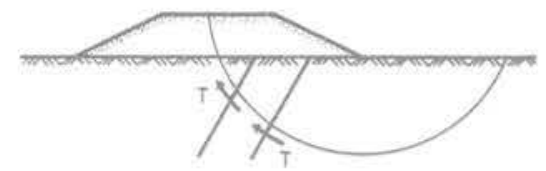

Fig. 13 Goujonnage d'un cercle de glissement profond

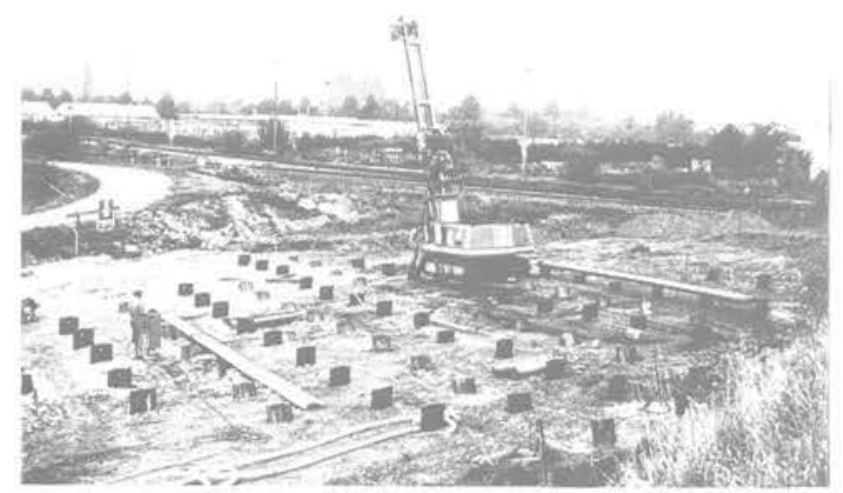

Fig. 14 Chantier d'essai avec armatures enfoncées à travers une couche superficielle

\subsection{Argiles surconsolidés}

On a attendu de nouveau les mécanismes de ruine de la figure 3 lors du clouage des sols argileux. Bien entendu, la cohésion doit apparaître dans le calcul de stabilité. A la différence du sable, la viscosité du sol peut être importante. De plus, des efforts tranchants dans les armatures (cf. fig. 13) peuvent jouer un rôle. Par manque d'une théorie consistante, tous les effets ont été étudiés par des expériences.

On a exécuté trois essais à grande échelle. Il fut assez difficile de trouver des couches uniformes suffisamment épaisses; lors de I'un des trois essais seulement ce fut exactement le cas. Le dispositif était à nouveau celui de la figure 7 , la surcharge d'un essai s'étendant jusqu'au bord du revêtement. La figure 15 donne une impression globale; on voit aussi que les déblais latéraux non renforcés ne sont pas stables. Pendant l'exécution on a eu quelques problèmes, ce qui n'est pas surprenant, avec la pluie et le froid. Pour étudier le fluage du sol, la surcharge fut maintenue constante dans diverses périodes de temps.

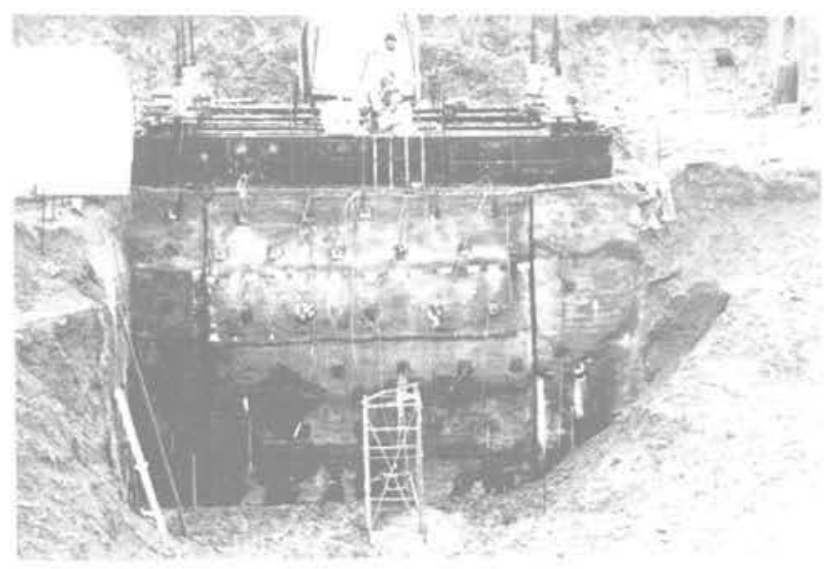

Fig. 15 Vue totale d'un essai à grande échelle dans une argile

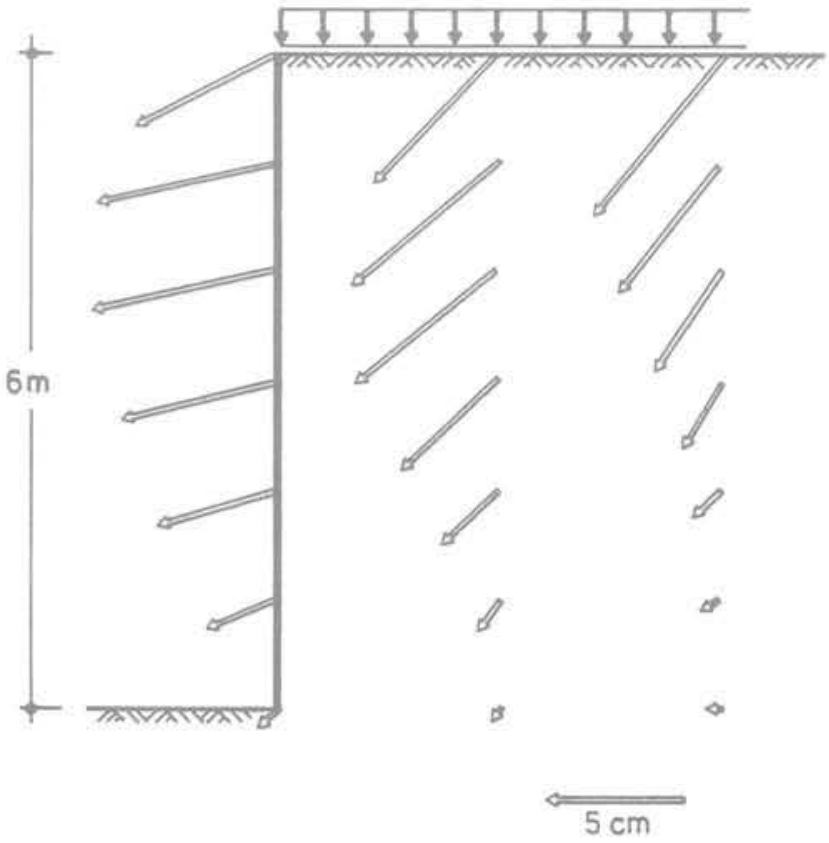

Fig. 16 Déplacements mesurés dans un massif argileux renforcé en état de service 
La figure 16 montre un champ de déplacement mesuré dans un état sûr (Schwarz 1980). La déformation moyenne est plus forte que celle du sable et se produit à volume constant. De telles conditions "non drainées" furent maintenues pendant tout l'essai. Les déformations croissent sous charges constantes au cours du temps comme le montre la figure 17. L'abaque de fluage est linéaire dans un diagramme bi-logarithmique, sa pente provenant seulement des caractéristiques du sol.

Dans le cas d'une surcharge jusqu'au bord, l'état limite est celui de Coulomb. On peut calculer les forces agissant sur le prisme glissant (fig. 18), à savoir : poids $W$, surcharge résultante $P$, force de cohésion $C$ (calculée avec la cohésion non drainée), force $\mathrm{A}$ selon l'équation 1. Ces forces sont en équilibre avec la force normale à la surface de glissement $\mathrm{N}$, si l'on ajoute une petite force tranchante $\mathrm{T}$ (fig. 18b). La dernière force doit être portée par les armatures. Sans doute, l'effet de goujonnage est faible ici et peut être négligé pour le dimensionnement.

Les distributions des efforts axiaux mesurées le long des armatures étaient analogues à celles de la figure 10 , la pente maximale cependant étant plus faible que pour le sable. Les pressions des terres avaient une distribution analogue à celles de la figure 11. Le rôle de la cohésion pour ces dernières n'a pas encore pu être clarifié.

\subsection{Argiles fissurées}

Quelques projets de clouage ont été réalisés par la Société Bauer dans des sols argileux avec des fissures. A titre d'exemple un cas est traité ici brièvement. Le sol était une marne rouge de la formation Keuper. Les caractéristiques sont bien connues par des essais triaxiaux avec échantillons de $0,6 \mathrm{~m}$ de diamètre (Wichter 1980). Dans un terrain faiblement incliné une paroi presque verticale de $16 \mathrm{~m}$ de profondeur maximale et d'environ $100 \mathrm{~m}$ de longueur devait être stabilisée à long terme. Les armatures de $8 \mathrm{~m}$ de longueur maximale étaient scellées avec des injections et protégées contre la corrosion.

L'arrangement des clous avait été préalablement dimensionné par des calculs simples de stabilité (correspondant aux fig. 4 et 18). Les forces d'arrachement, les pressions des terres et les déformations avaient été estimées à l'aide des essais à grande échelle. Dans trois profils des installations avaient été placées pour mesurer les déformations du sol et les forces axiales dans les armatures. Après ces travaux préalables, le gouvernement a donné son autorisation.

Le clouage a été exécuté sans problème; la figure 19 donne une impression des travaux. Les déformations furent très petites $(u \leqslant 0,0015 h)$ avec une faible composante de fluage. Les forces d'arrachement des clous furent plus grandes que prévues. Le clouage était nettement plus économique dans ce cas que les autres méthodes de soutènement (mur ancré, rangée de pieux, etc.).

\section{Développements récents}

\subsection{Nouvelles variantes du clouage}

Le clouage peut être utilisé pour la reprise en sous-œuvre directement à côté de bâtiments déjà existants. C'est possible dans le cas des sols

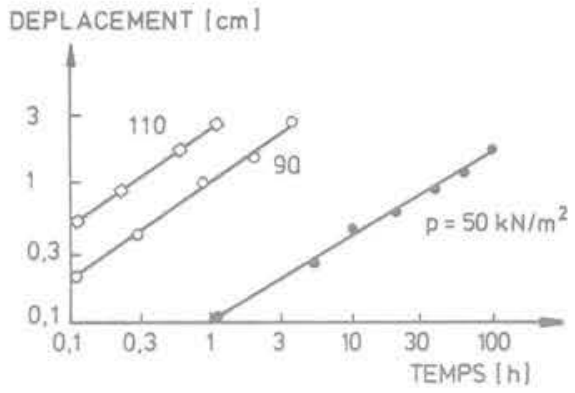

Fig. 17 Déplacements horizontaux au bord du revêtement soumis à différentes charges constantes

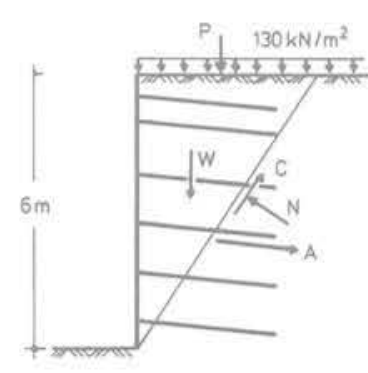

a)

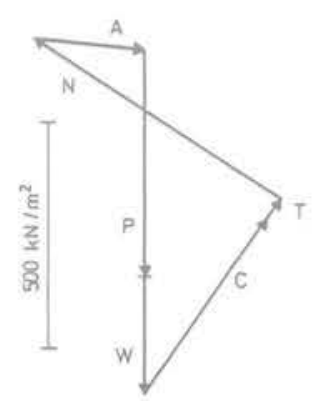

b)
Fig. 18 Etat limite atteint lors d'un essai à grande échelle dans de l'argile

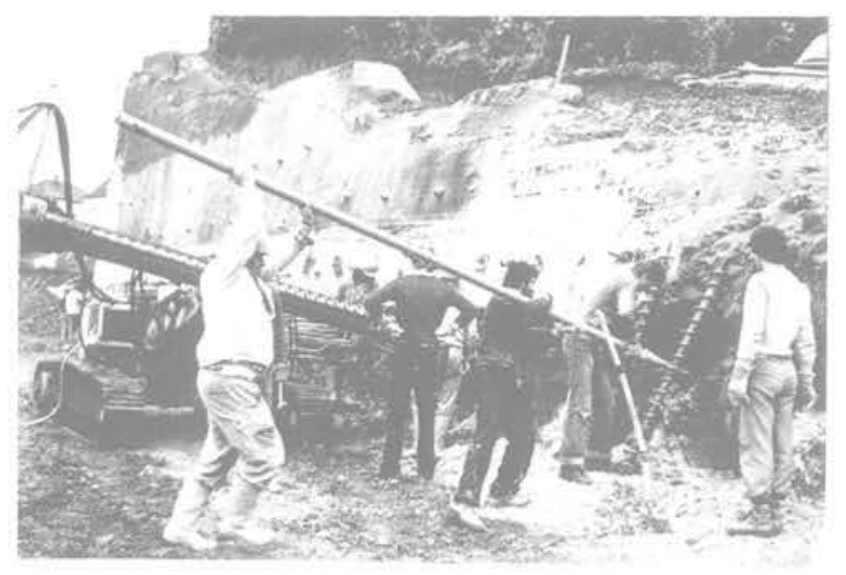

Fig. 19 Cloutage à long terme d'une marne rouge

granulaires ou argileux cimentés parce qu'ils cèdent très peu jusqu'à ce qu'une mobilisation suffisante des efforts dans les armatures soit atteinte. Bien entendu, on doit excaver avec beaucoup de prudence. La cohésion doit être partiellement augmentée par injection. II est adéquat que le revêtement s'étende toujours plus profondément que l'excavation.

Le clouage du type de la figure $2 a$ peut être réalisé par des pieux (ou mieux des goujons) de sections différentes. A présent, trois types d'armatures se compcsant d'acier et de ciment avec des diamètres de $12 \mathrm{~cm}$ à $60 \mathrm{~cm}$ sont installés dans des talus d'argile. On mesure les déformations du sous-sol et les efforts dans quelques pieux. 


\subsection{Méthodes théoriques nouvelles}

Pour simplifier le dimensionnement du clouage d'un déblai on a développé des abaques (Gässler et Gudehus 1981). Les paramètres du système peuvent être variables selon la figure 20 (b est la distance horizontale des clous). On a fait varier le mécanisme composé pour trouver le facteur de sécurité minimale $\min \eta$. Pour chaque ensemble de variables $\lambda=\frac{\ell_{N}}{h}$ (longueur relative des clous), $\alpha, \beta, \varepsilon$ et $\rho$ on obtient une abaque donnant $\min \eta$ et la pente $\theta$ du plan de glissement inférieur. Un exemple se trouve sur la figure 21, et d'autres seront donnés par Gässler (1982). Le paramètre décisif est la résistance spécifique des clous, définie par

$$
\mu=T_{m} / \gamma a b .
$$

Le calcul de $\eta$ n'est pas complètement objectif parce que le choix de la force fictive $T$, est partiellement arbitraire (voir paragraphe 2.1). Ce défaut peut être surmonté par le calcul statistique de la sécurité donné par l'Eurocode l. C'est le but d'un projet de recherche à Karlsruhe que d'unifier cette méthode nouvelle et la méthode bien connue des facteurs partiels de sécurité.

D'autres méthodes ont été développées pour le clouage du type des figures $2 \mathrm{a}$ et $\mathrm{b}$. On peut calculer l'interaction du sol avec les armatures en tenant compte du fluage et du durcissement du sol (Winter 1980).
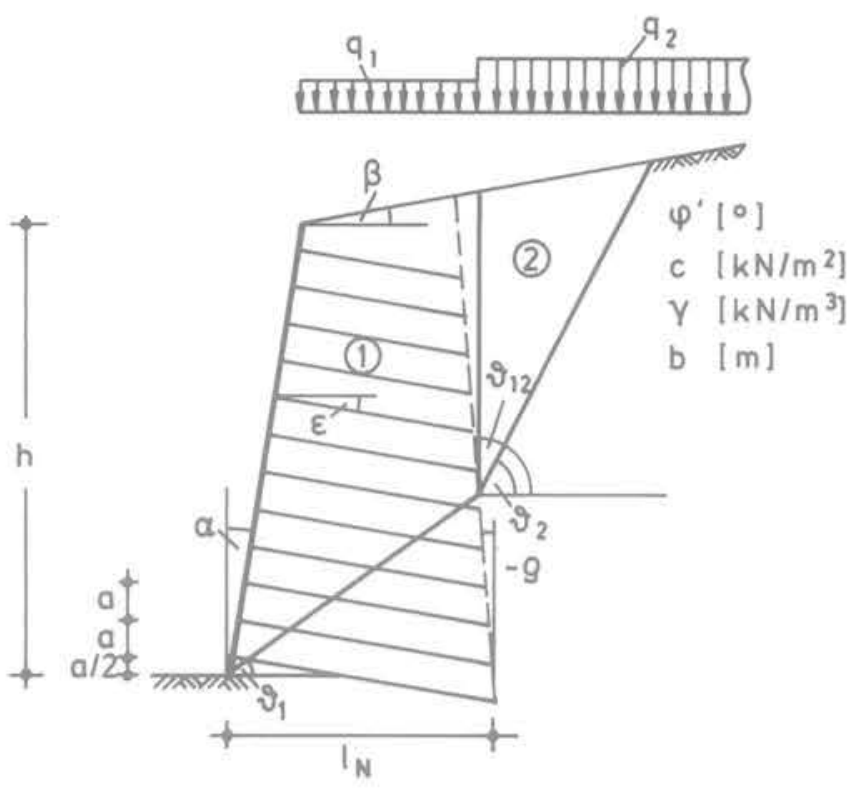

Fig. 20 Paramètres pour le dimensionnement par abaques

\section{Conclusion}

Le renforcement du sol in situ, appelé clouage, peut être sûr et économique. Des règles de dimensionnements pour des sols granulaires ont été développées et vérifiées par 4 essais à grande échelle. La stabilité globale peut être calculée exactement par des mécanismes composés de translation. Les efforts existants dans le revêtement et les clous peuvent être estimés sur la base des lois de similitude. Les déformations sont petites, même si une partie du chargement est cyclique.

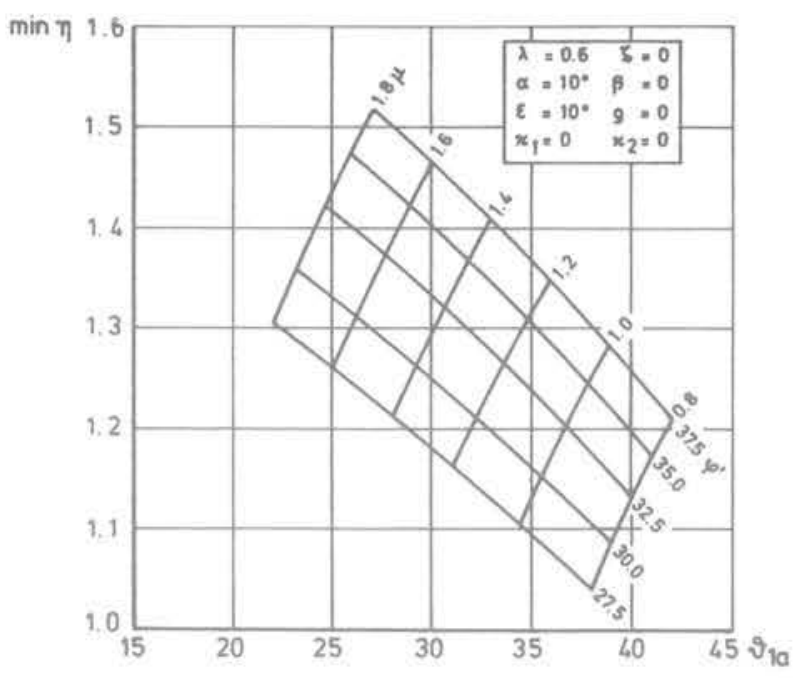

Fig. 21 Abaque de dimensionnement (exemple)

Pour les sols argileux, la stabilité globale peut être calculée à l'aide de méthodes analogues, Dans des sols mous, les armatures supportent des efforts tranchants; ainsi un goujonnage des cercles de glissement est obtenu. Par 2 essais à grande échelle on a pu démontrer cet effet. Pour des déblais dans des sols raides, les armatures supportent des forces axiales. Dans 3 essais à grande échelle, les mécanismes de ruine étaient les mêmes que dans le sable. Les déformations peuvent être plus grandes et en outre augmenter par fluage. Dans le cas des sols argileux fissurés (par exemple marne rouge), les déformations sont plus petites; cela a été démontré par quelques projets réels.

On peut utiliser le clouage à côté de bâtiments existants. Le goujonnage de 3 versants argileux par des pieux est actuellement étudié in situ. Les méthodes de calcul du clouage ont encore été développées en tenant compte des paramètres statistiques et du fluage.

\section{Références bibliographiques}

G. Gässler (1978). Large Scale Dynamic Test of in situ Reinforced Earth. Proceed. Dynam. Meth. Soil and Rock Mech., Vol. 2, Balkema, Rotterdam, p. 333.

G. Gässler et G. Gudehus (1981). Soil Nailing - Some Aspects of a New Technique. Proceed Xth Intern. Conf. Soil Mech. Found. Eng. Stockholm, pp. 665-670.

G. Gässler (1982). Tragverhalten und Standsicherheit vernagelter Wände und Böschungen. Ver̈offentlichungen des Instituts für Bodenmechanik und Felsmechanik, Karlsruhe, Heft 90.

M. Goldscheider et D. Kolymbas (1980). Berechnung der Standsicherheit mehrfach verankerter Stützwände. Geotechnik, Jahrgang 3, Heft 4, pp. 156-164.

G. Gudehus (1972). Lower and upper bounds for stability of earth-retaining structures. Proceed. 5th Europ. Conf. Soil Mech. Found. Engg., Mai 1972, Vol. 1, Madrid, pp. 21-28. 
G. Gudehus (1980). Vernagelung von Böschungen und im Dammuntergrund. Schriftenreihe Erd- und Grundbau, Heft 3, Forschungsges. Straßenwesen, Köln.

G. Gudehus (1980a). Materialverhalten von Sand: neuere Erkenntnisse. Bauingenieur 55, pp. 57-67.

G. Gudehus (1980 b). Materialverhalten von Sand: Anwendung neuerer Erkenntnisse im Grundbau. Bauingenieur 55, pp. 351-359.

G. Gudehus (1981). Bodenmechanik. Enke, Stuttgart.

Louis C. (1981). Nouvelle méthode de soutènement des sols en déblais. Travaux No. 553, Mars 1981, pp. 67-75.

W. Schwarz (1980). Rapport de recherche sur le cloutage des sols argileux.
M. Stocker et G. Gässler (1979). Ergebnisse von Grossversuchen über eine neuartige BaugrubenwandVernagelung. Der Tiefbau, Sept. 1979, pp. 677-686.

M. Stocker, G.W. Körber, G. Gässler et G. Gudehus (1979). Soil Nailing. Comptes Rendus, Colloque International sur le renforcement des sols, Paris, pp. 469-474.

L. Wichter (1980). Festigkeitsuntersuchungen an Grossbohrkernen von Keupermergel and Anwendung auf eine Böschungsrutschung. Veröffentl. Institut für Bodenmechanik und Felsmechanik, Karlsruhe, Heft 84.

H. Winter (1980). Bemessung von Pfahlgründungen und Hangverdübelungen auf Fließdruck. Vortrảge der Baugrundtagung Mainz, Deutsche Gesellschaft f. Erdund Grundbau. 
